\title{
Numerical prediction for the performance of a floating-type breakwater by using a two-dimensional particle method ${ }^{\dagger}$
}

\author{
Byung-Hyuk Lee ${ }^{1}$, Sung-Chul Hwang ${ }^{2}$, Jung-Woo Nam ${ }^{2}$ and Jong-Chun Park ${ }^{2}$ \\ ${ }^{1}$ Dept. of Ocean Industry Research, Hyundai Maritime Research Institute, Ulsan, South Korea \\ ${ }^{2}$ Dept. of NAOE, Pusan National University, Busan, 609-735, South Korea
}

(Manuscript Received December 14, 2010; Revised January 11, 2011; Accepted February 7, 2011)

\begin{abstract}
The nonlinear free-surface motions interacting with a floating body were investigated using the Moving Particle Semi-implicit (MPS) method proposed by Koshizuka and Oka [6] for incompressible flow. In the numerical method, more realistic Lagrangian moving particles were used for solving the flow field instead of the Eulerian approach with a grid system. Therefore, the convection terms and time derivatives in the Navier-Stokes equation can be calculated more directly, without any numerical diffusion, instabilities, or topological failure. The MPS method was applied to a numerical simulation of predicting the efficiency of floating-type breakwater interacting with waves.
\end{abstract}

Keywords: Floating-type breakwater, Wave transmission rate, Particle method, Navier-Stokes equation, Waves interacting with a floating body

\section{Introduction}

In order to efficiently utilize the coastal area, various kinds of breakwater should be studied and developed. Although fixed breakwaters have excellent performance onshore, they are associated with economical and technical problems in their construction offshore, as well as environmental by restricting the circulation of seawater. However, floating-type breakwaters have many advantages compared to fixed ones, i.e. flexibility of future extensions, mobility, preservation of environments and economical efficiency, etc. As a result, a few investigations have proposed to improve the performance of floating breakwaters $[1,2,3,5,8$, $11,12,14]$. Of these, most numerical approaches relating to floating breakwaters have focused on developing numerical techniques that capture the fully nonlinear free-surface motion based on a grid system.

\footnotetext{
†This paper was presented at the 2nd International Symposium on Shallow Flows(ISSF), Hong Kong, December 2008.

*Corresponding author. Tel.: +82 51510 2480, Fax.: +82 515832485 .

E-mail address: jcpark@pnu.edu

Copyright (C) KSOE 2011.
}

However, there are many different approaches that do not employ a grid system; for example, the so-called particle methods with a fully Lagrangian treatment [6, 10]. The particle methods seem to be more feasible and effective than conventional grid methods for solving the flow fields associated with complicated boundary shapes or coupling effects between a fluid and structure.

In the present study, the efficiency of a floating-type breakwater interacting with waves was investigated numerically, using the Moving Particle Simulation (MPS) method supposed by Koshizuka and Oka [6] for an incompressible flow. In this method, more realistic Lagrangian moving particles were used for solving the flow field rather than an Eulerian approach with a grid system. Therefore, the convection terms and time derivatives in the Navier-Stokes equation can directly be calculated, without any numerical diffusion, instability or topological failure. The method consisted of particle interaction models to represent the gradient, diffusion, incompressibility and free-surface boundary conditions. 


\section{Moving particle simulation}

\subsection{Governing function}

The governing equations for incompressible viscous flows are the continuity and Navier-Stokes equations, as follows:

$$
\begin{aligned}
& \frac{D \rho}{D t}=0 \\
& \frac{D \vec{u}}{D t}=-\frac{1}{\rho} \nabla P+\nu \nabla^{2} \vec{u}+\vec{F}
\end{aligned}
$$

where $\rho$ is the density, $t$ the time, $\vec{u}$ the velocity vector, $\nabla$ the gradient, $P$ the pressure, $v$ the kinematic viscosity and $\vec{F}$ the external force.

The left-hand side of the Navier-Stokes equation (2) denotes a Lagrangian differentiation, which is directly calculated by moving particles in a Lagrangian manner. The right-hand side consists of the pressure gradient, viscous and external force terms. To simulate incompressible flows, all terms expressed by differential operators should be replaced by the particle interaction models of the MPS method.

\subsection{Kernel function}

Continuous fluid can be represented by physical quantities of coordinates, mass, velocity components and pressure for particles. The governing equations written with partial differentiations are transformed to the equation for particle interactions. The particle interactions in the MPS method are based on a kernel function. In this study, the following function was employed.

$$
w(r)=\left\{\begin{array}{cc}
\frac{r_{e}}{r}-1 & \left(0 \leq r<r_{e}\right) \\
0 & \left(r_{e}<r\right)
\end{array}\right.
$$

where the distance between two particles is given by $r$, and $r_{e}$ represents the effective range of the particle interactions. In Fig. 1, the kernel becomes zero when $r>r_{e}$. Since the area covered by this weight function is bounded, a particle interacts with a finite number of neighboring particles. The radius of the area of interaction is determined by a parameter, $r_{e}$.

The weighting of an interaction between two particles can be described by a kernel function, i.e. the nearer the distance between two particles, the larger the weight of the interaction. If the distance between two particles is quite long, the weight of the interactions can be neglected.

\subsection{Gradient model}

A gradient vector between two particles, $i$ and $j$, possessing scalar quantities, $\phi_{i}$ and $\phi_{j}$, at coordinates, $r_{i}$ and $r_{j}$, is simply defined by $\left(\phi_{j}-\phi_{i}\right)\left(r_{j}-r_{i}\right) /\left|r_{j}-r_{i}\right|^{2}$, as shown in Fig. 2. The gradient vector at the particle $i$ is given by the weighted average of the gradient vectors:

$$
\langle\nabla \phi\rangle_{i}=\frac{d}{n^{0}} \sum_{i \neq j}\left[\frac{\phi_{j}-\phi_{i}}{\left|r_{j}-r_{i}\right|^{2}}\left(\vec{r}_{j}-\vec{r}_{i}\right) w\left(\left|\vec{r}_{j}-\vec{r}\right|\right)\right]
$$

where $d$ is the number of space dimensions, and $n^{0}$ the particle number density, which is fixed for incompressibility under the initial condition of the particle arrangement. The particle number density is calculated by the following equation.

$$
n_{i}=\sum_{i \neq j} w\left(\left|\vec{r}_{j}-\vec{r}_{i}\right|\right)
$$

The fluid density is proportional to the particle number density.

\subsection{Diffusion model}

The diffusion of $\phi$ at the particle $i$ is described

by

$$
\begin{aligned}
& \nabla^{2} \phi=\frac{2 d}{\lambda}\left(\phi_{j}-\phi_{i}\right) w\left(\left|r_{j}-r_{i}\right|\right) \\
& \lambda=\frac{\sum_{j \neq i} w\left(\left|\vec{r}_{j}-\vec{r}_{i}\right|\right)\left|r_{j}-r_{i}\right|^{2}}{\sum_{j \neq i} w\left(\left|\vec{r}_{j}-\vec{r}_{i}\right|\right)} \cong \frac{\int_{V} w(r) r^{2} d v}{\int_{V} w(r) d v}
\end{aligned}
$$

where $\lambda$ is a parameter where the increase in the variance is equal to that of the analytical solution.

The diffusion can be modeled by the distribution of a physical value from a particle to its neighboring particles using the kernel function (Fig. 3). The model is conservative, since the quantity lost by the particle, $i$, is only obtained by the neighboring particles, $j$. 


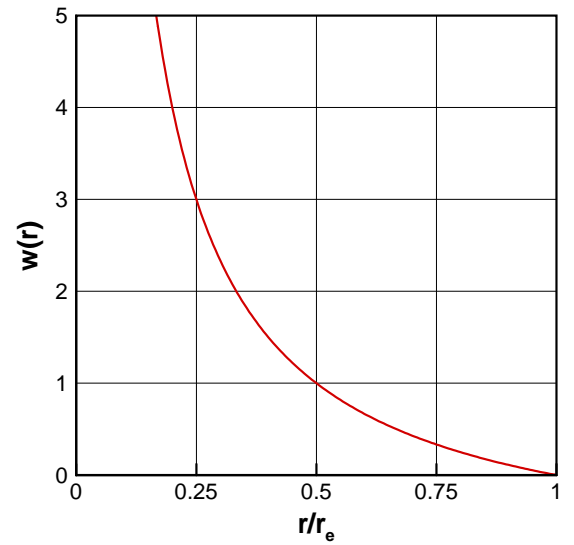

Fig. 1 Kernel function.

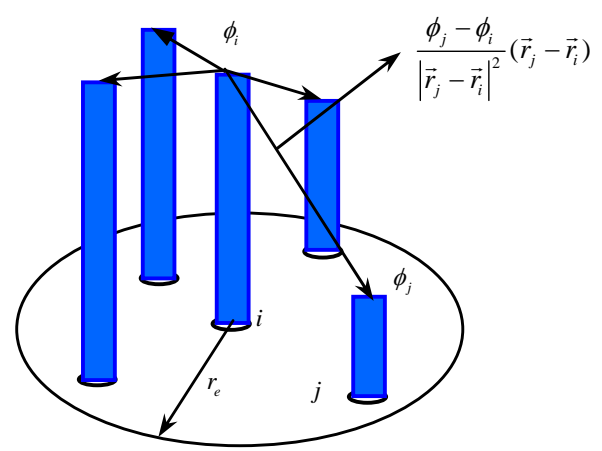

Fig. 2 Gradient model.

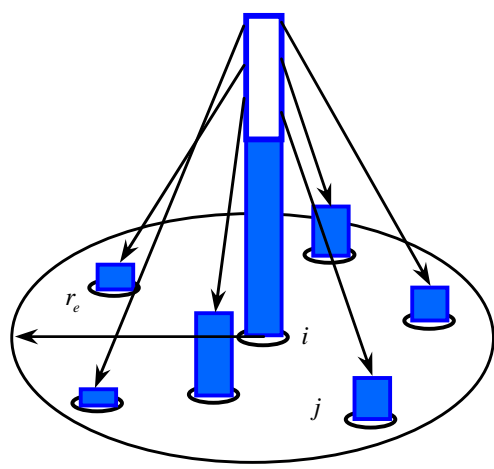

Fig. 3 Diffusion model.

\subsection{Incompressibility model}

The fluid density is represented by the particle number density. Thus, the continuity equation (1) is fulfilled by fixing the particle number density via simulation. This means that the particle number density, $n^{0}$, should be constant.

The algorithm for incompressibility in the MPS method is similar to that in the SMAC (Simplified Marker-and-Cell) method with a grid system. There are two stages in each time step: in the first stage, the temporal velocity components and coordinates of the particle $i$ are obtained using diffusion, external forces and convection terms, which are explicitly calculated with the values in the (n)-th time step. Thus the temporal coordinates $\vec{r}_{i}^{*}$ of the particle $i$ can be written using the temporal velocity $\vec{u}_{i}^{*}$, as follows:

$$
\vec{r}_{i}^{*}=\vec{r}_{i}^{n}+\Delta t \vec{u}_{i}^{*}
$$

In equation (8), the convective term can be simply calculated via the moving particles according to the temporal velocity components, $\vec{u}_{i}^{*}$. Due to the movement of particles in the explicit first stage, the particle number densities might be changed, i.e. $n^{*} \neq n^{0}$.

In the second stage, the temporal particle number densities, $n_{i}^{*}$, are calculated from the temporal coordinates, $\vec{r}_{i}^{*}$. The Poisson equation for a pressure is calculated implicitly [6]:

$$
\left\langle\nabla^{2} P\right\rangle_{i}=-\frac{\rho}{\Delta t^{2}} \frac{n_{i}^{*}-n^{0}}{n^{0}}
$$

The right hand side of equation (9) represents the deviation of the temporal particle number density from a constant value. The role of the right hand side in equation (9) maintains the particle number densities during the simulation. The left hand side of equation (9) is discretized by the diffusion model (6). Finally, simultaneous equations expressed by a linear symmetric matrix are obtained, which are solved using an iteration method. In the present study, the CG (Conjugate Gradient) method was employed as the iterative solver.

Fig. 4 shows the algorithm procedure for the present method. After updating the pressure field, the velocity correction $\vec{u}_{i}$ is calculated by the following equation:

$$
\vec{u}_{i}^{\prime}=-\frac{\Delta t}{\rho}\left\langle\nabla P^{n+1}\right\rangle
$$

Finally, the velocity components and coordinates of particles in the $(n+1)$-th time step are calculated from the following equations:

$$
\begin{aligned}
& \vec{u}_{i}^{n+1}=\vec{u}_{i}^{*}+\vec{u}_{i}^{\prime} \\
& \vec{r}_{i}^{n+1}=\vec{r}_{i}^{*}+\Delta t \vec{u}_{i}^{\prime}
\end{aligned}
$$




\subsection{Boundary condition}

As the free-surface boundary condition, the kinematic and dynamic boundary conditions are imposed. The kinematic condition can be directly satisfied by moving particles on the free-surface. In the present method, it was straightforward to track the freesurface particles, because the location of the freesurface was easily obtained as a result of the fully Lagrangian treatment of the particles.

As shown in Fig. 5, in the vicinity of the freesurface, the particle number densities were decreased, as the empty air region, where no particles exist in the case of single-phase problem, was included. Thus, the particles satisfying the following simple condition were considered on the free surface.

$$
\langle n\rangle_{i}^{*}<\beta n^{0}
$$

where $\beta$ is a parameter below $1.0 ; \beta=0.97$ was selected in this study. Using this free-surface boundary condition, the simulation of fragmentation and coalescence of a fluid is available. The free-surface parameter, $\beta$, was used to judge whether the particles were on the free-surface.

Conversely, the dynamic condition can be satisfied by taking the atmospheric pressure ( $\left.P=P_{a t m}=0\right)$ on the free-surface particles. This condition was fulfilled in the procedure of solving the Poisson equation (9).

For the wall boundary condition, as shown in Fig. 6, the wall particles were set according to the solid boundary, with dummy particles inside the solid wall. In the particle method, it is important to obtain useful information on the physical quantities from the neighboring particles. The physical quantities were calculated by the interaction with neighboring particles. The wall particles will be directly in contact with both the fluid and dummy particles, which are involved in the pressure correction calculation and prevent the concentration of particles near the wall. They also have zero velocities when the walls are treated as non-slip. The dummy particles contain velocity components, which are installed in the same way as the dummy cells in grid methods. Three layers of particles are located to ensure that the particle number density is accurately computed.

\subsection{Treatment of passively moving solid model}

In this section, two numerical treatments have been introduced for solving the motion of a floating body; one being the conventional method using the equation

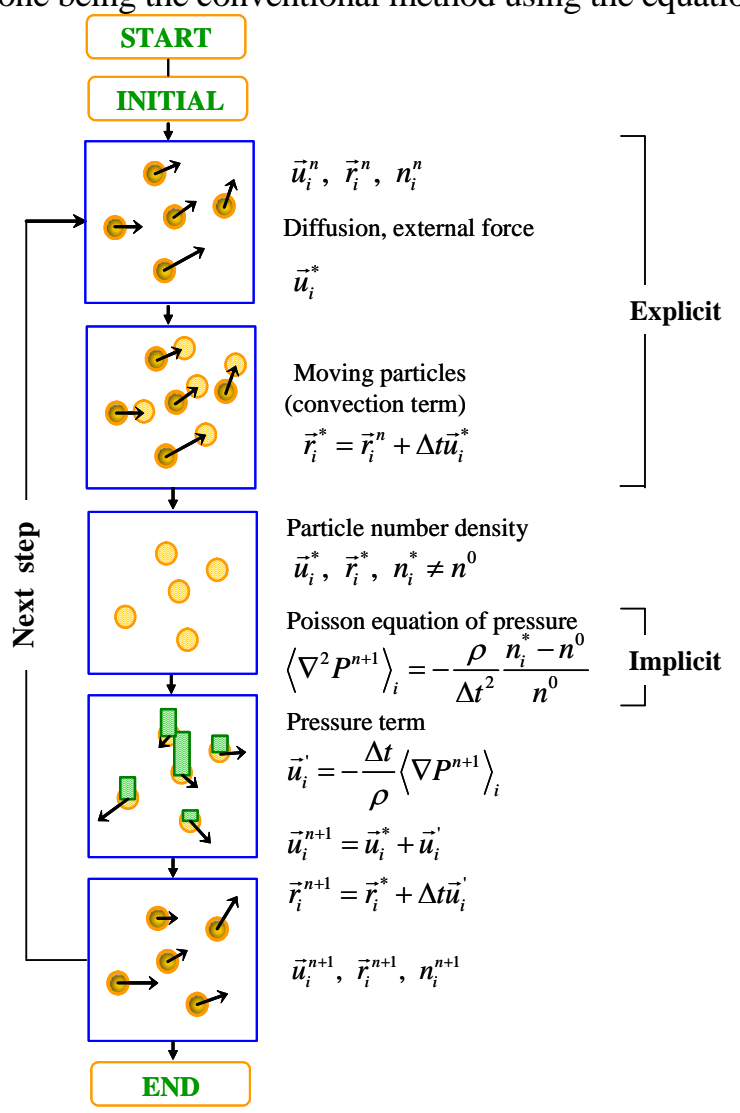

Fig. 4 Algorithm procedure of MPS.

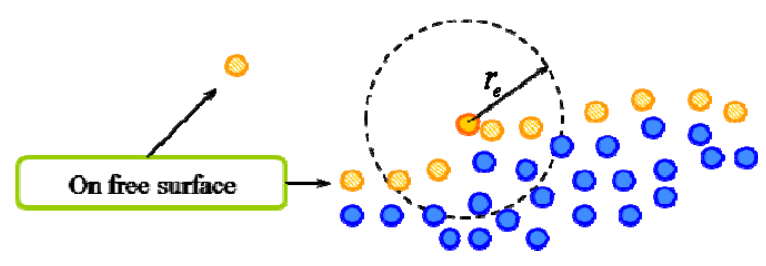

Fig. 5 Free-surface boundary condition.

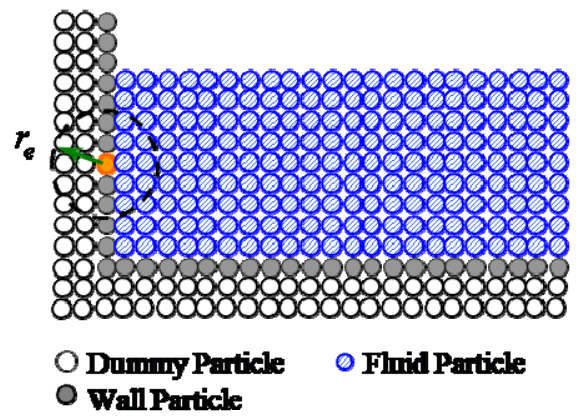

Fig. 6 Wall boundary condition.

of motion, and the other is the passively moving-solid model suggested by Koshizuka and Oka [6], which describes the motion of a rigid body in a fluid. 
Sueyoshi [13] proposed another method, which solves the equation of motion explicitly, to determine the motion of a rigid body in a fluid. The force and moment acting on the surface of a rigid body are directly calculated by the integration of the pressure on its surface, as shown in Fig. 7. The equation of motion for a floating body in 2-dimension can be calculated through the translation and rotational angles of a floating body.

$$
\begin{aligned}
& m \frac{d^{2} \vec{r}_{g}}{d t^{2}}=\vec{F} \\
& I_{z z} \frac{d^{2} \theta}{d t^{2}}=\vec{M} \cdot \vec{k}
\end{aligned}
$$

Here, $m$ is mass of the solid, $\vec{r}_{g}$ the center of gravity, $\theta$ the rotational angle for rotational axis, $I_{z z}$ the moment of inertia for rotational axis and $\vec{k}$ the unit vector normal to the 2-dimensional plane. The hydrodynamic force, $\vec{F}$, and moment, $\vec{M}$, affect the translational and rotational motions, respectively, and are calculated via:

$$
\begin{aligned}
& \vec{F}=\sum_{i}^{\text {on the hull surface }} P_{i} \vec{n}_{i} d S_{i} \\
& \vec{M}=\sum_{i}^{\text {on the hull surface }}\left(\vec{r}_{i}-\vec{r}_{g}\right) \times P_{i} \vec{n}_{i} d S_{i}
\end{aligned}
$$

Here, $\vec{n}_{i}$ and $d S_{i}$ are the normal vector and the local area of a particle on the surface of a rigid body, respectively.

Conversely, Koshizuka and Oka [6] proposed a passively moving-solid model to describe the motion of a rigid body in a fluid. Here, a solid is assumed to be a collection of particles held together by intermolecular forces. The solid particles are initially calculated using the same incompressible algorithm as for fluid particles. At this stage, the coupling effect between the individual solid particles is not considered. As a result of the simulation, at this stage the solid deforms; therefore the relative locations of the solid particles should be corrected using the equations (18) (23).

At the center of a rigid body, the translational velocity, $\vec{T}$, and rotational velocity, $\vec{R}$, are calculated as given in the following equations:

$$
\vec{T}=\frac{1}{n} \sum_{i=1}^{n} \vec{u}_{i}
$$

$$
\vec{R}=\frac{1}{I} \sum_{i=1}^{n} \vec{u}_{i} \times \vec{q}_{i}
$$

Here, the relative coordinates between solid particles, $\vec{q}_{i}$, and the moment of inertia, $I$, are given as:

$$
\begin{aligned}
& \vec{q}_{i}=\vec{r}_{i}-\vec{r}_{g} \\
& I=\sum_{i=1}^{n}\left|\vec{q}_{i}\right|^{2}
\end{aligned}
$$

Here, $\vec{r}_{g}$ is the center of gravity, which is calculated as follows:

$$
\vec{r}_{g}=\frac{1}{n} \sum_{i=1}^{n} \vec{r}_{i}
$$

Finally, the velocity vector of the solid particles was replaced by:

$$
\vec{u}_{i}=\vec{T}+\vec{q}_{i} \times \vec{R}
$$

In the next time step, the fluid particles are slightly affected by this solid motion via an incompressibility calculation.

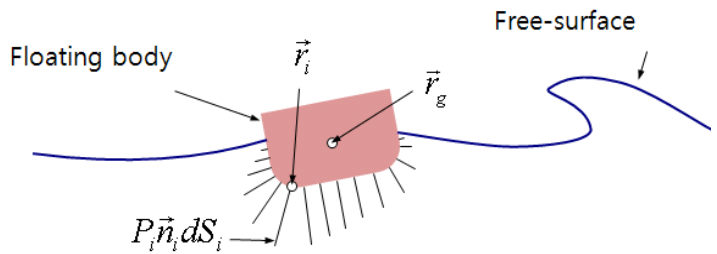

Fig. 7 Schematic of motion equation for 2D floating body simulation.

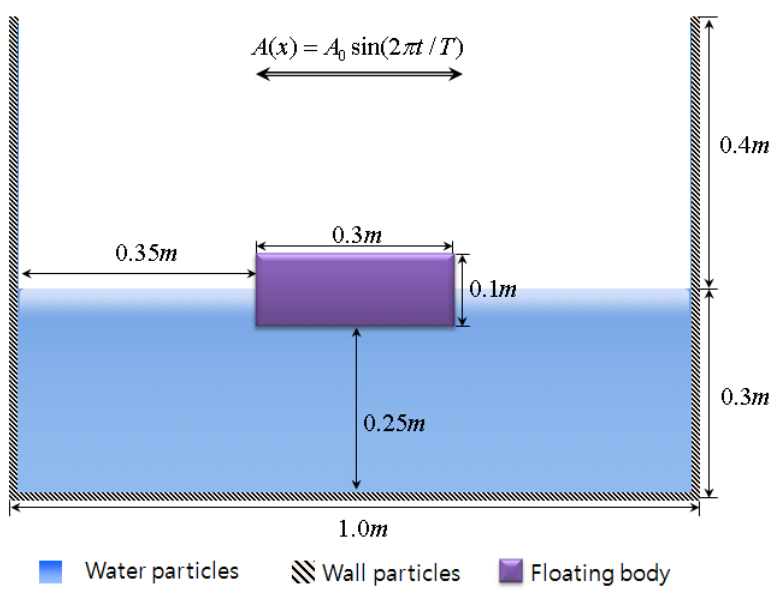

Fig. 8 Initial configuration of 2D floating body simulation. 


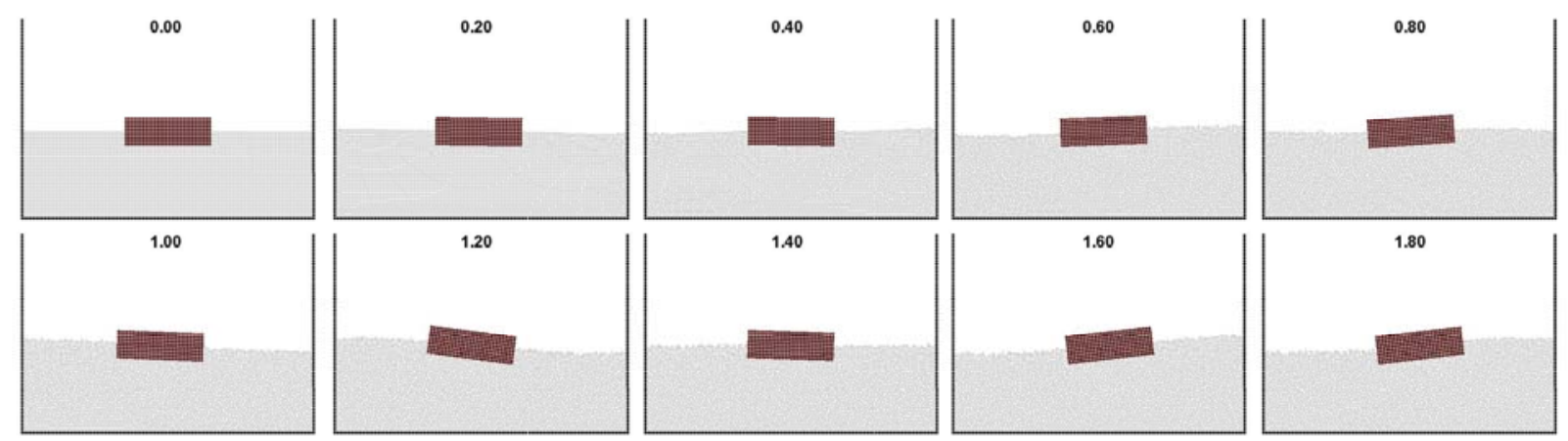

(a) Moving solid model
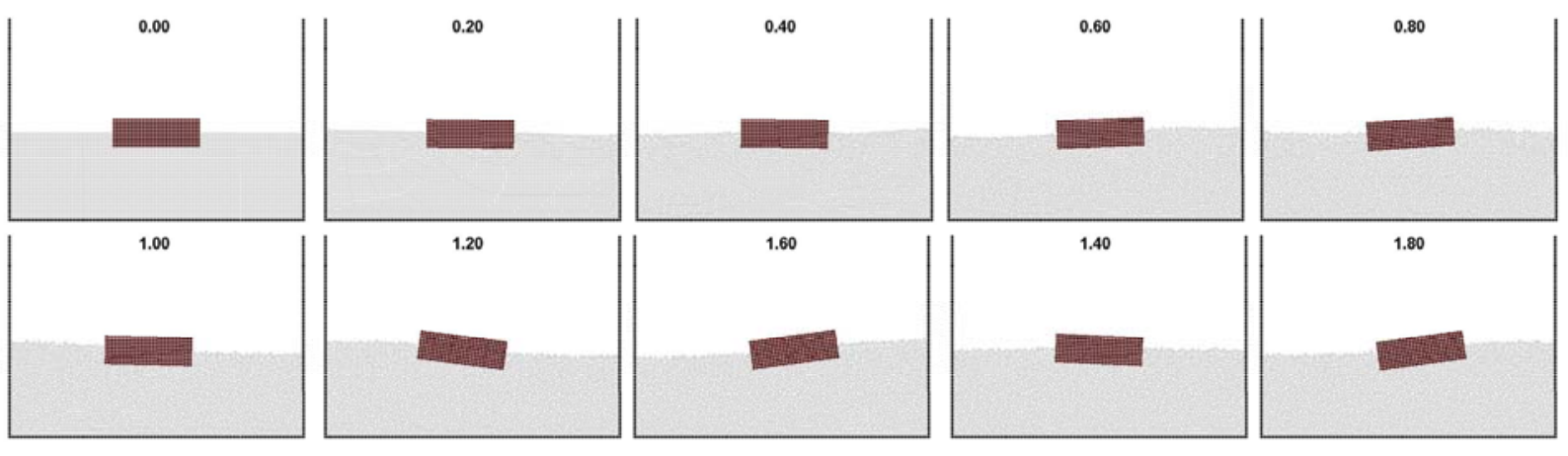

(b) Equation of motion

Fig. 9 Floating body motion of coupled with free-surface motion.

\section{Numerical simulation and discussions}

\subsection{Floating-body simulation}

To verify the simulation of a floating body with free-surface, the motions of a 2D floating rectangular barge, in an oscillating water-filled (density $=1000$ $\mathrm{kg} / \mathrm{m}^{3}$ ) tank, are simulated in the time domain using both the equation of motion and the passively moving-solid model.

The initial geometry and set-up are shown in Fig. 9. The height and width of the tank were 1.0 and $0.7 \mathrm{~m}$, respectively. The top of the tank was opened and the water depth was $0.3 \mathrm{~m}$. The floating body's height and width were 0.1 and $0.3 \mathrm{~m}$, respectively. The body was initially positioned in the center of the tank. The specific gravity and density of the body were 0.5 and $500 \mathrm{~kg} / \mathrm{m}^{3}$, respectively. The initial distance between particles, $l_{0}$, was $0.01 \mathrm{~m}$, with the total number of particles being 4,000. The movement of the tank followed a sinusoidal function (Eq. 24); therefore, a harmonic flow field was created in the tank.

$$
A(t)=A_{0} \sin (2 \pi t / T)
$$

Here, the amplitude $A_{0}$ and period $T$ of the movement were $0.01 \mathrm{~m}$ and $1.0 \mathrm{sec}$, respectively.

Fig. 9 shows snap-shots of the simulated results for the motion of the floating body interacting with a free-surface. Fig.10 (a) shows the results on application of the moving solid model; whereas, Fig.10 (b) shows the results for the equation of motion; both cases looked very similar.

Fig. 10 shows the corresponding time histories of the roll motions of the floating body. Both results are appeared to be very close to the anticipated period of 1sec. Due to the standing wave formed inside the tank, the amplitudes were modulated. The slight discrepancy between the two sets of results might have been caused by the different algorithm and the nonphysical pressure fluctuation in the pressure integration used for the equation of motion. From this comparison, the use of the passively moving-solid model was validated, which is a simpler algorithm and computationally more economical compared to the equation-of-motion method. For the ensuing examples shown in this paper, the passively moving solid model was employed to calculate the motion of a floating body. 


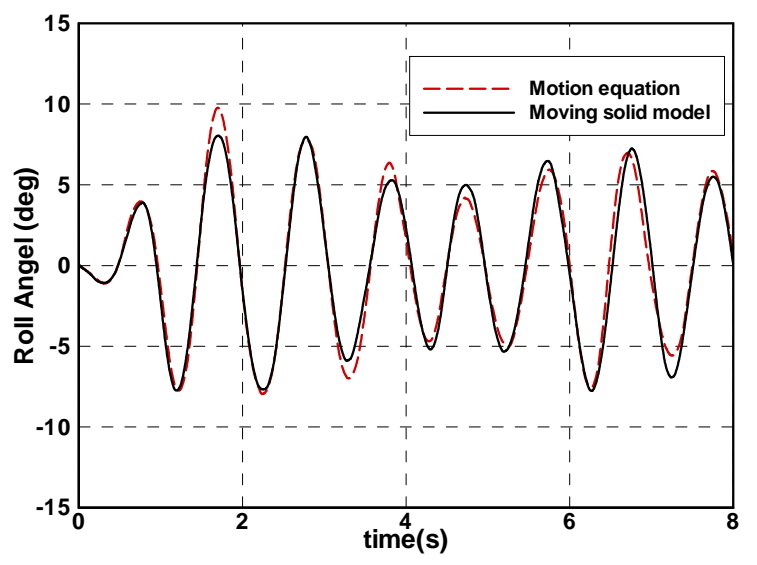

Fig. 10 Time history of roll angle of floating body.

\subsection{Numerical prediction on performance of float- ing-type breakwater}

The numerical predictions on the performance of a floating-type breakwater were performed. The initial geometry for the simulation is illustrated in Fig.11. The floating breakwater, fixed by a hinge to the bottom, was located in $2.0 \mathrm{~m}$ left from the piston-type wavemaker. The wave absorber was located to the right of the floating breakwater to absorb the wave made by the wavemaker. The viscous effects and surface tension were disregarded in this simulation. The floating breakwater was assumed to be rigid and composed of solid particles. The total simulation time was 20.0sec.

When the wave generated by the wavemaker had fully developed, the performance of the floating breakwater was computed. The movement of the piston-type wave-maker follows a sinusoidal function (24).

The period and length of the generated waves by oscillating wave-maker are shown in Table 1 . The generated wave height was $0.2 \mathrm{~m}$ with low and high tide levels of 0.82 and $0.2 \mathrm{~m}$, respectively. The total number of particles used for the simulation is about 25000. The densities of the floating breakwater and water were 500 and $1000 \mathrm{~kg} / \mathrm{m}^{3}$, respectively. The time increments were varied under the Courant's stability condition.

The wave height and length according to the time increments were measured at $5.0 \mathrm{~m}$ from the wavemaker, without a floating breakwater. The numerical prediction on the performance of the floating breakwater was performed after the wave conditions of the simulated results, without a floating body, were com- pared with the simulated conditions shown in Table 1. The performance of the breakwater at low and high tide levels were calculated via the measurement of the wave height at 4.0 and $6.0 \mathrm{~m}$ from the wavemaker. The transmissivity can be defined as:

$$
\text { Transmissivity }(\%)=\frac{\text { Output wave height }}{\text { Input wave height }} \times 100
$$

where the initial wave elevation without a body condition was considered.

Table 1 Principal parameter for wavemaker.

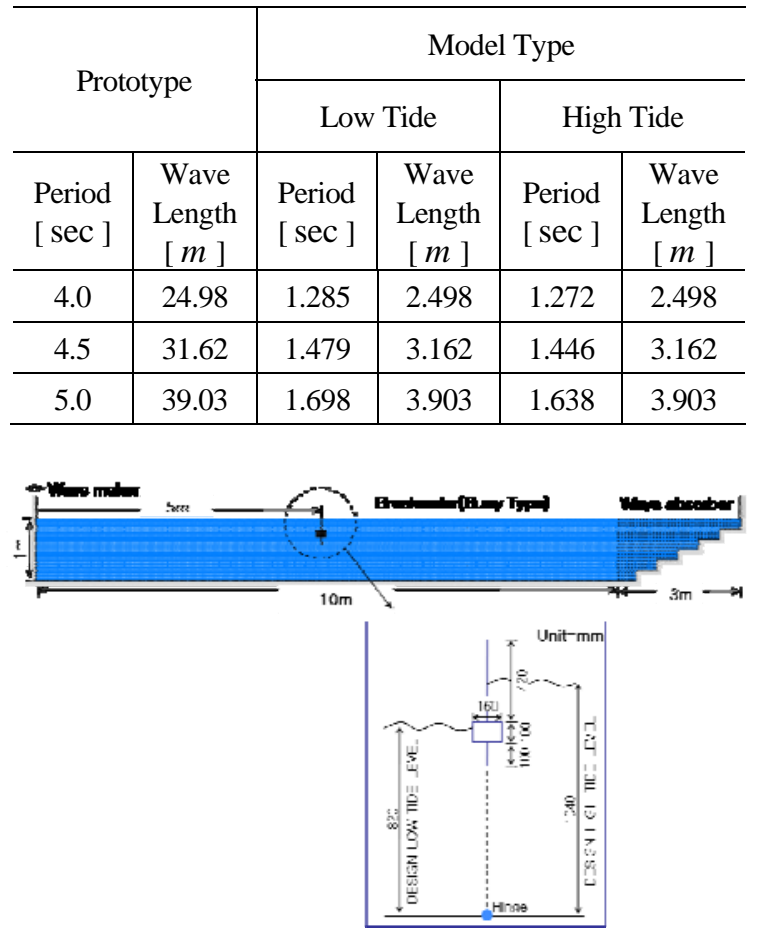

Fig. 11 Schematic of set up for breakwater.

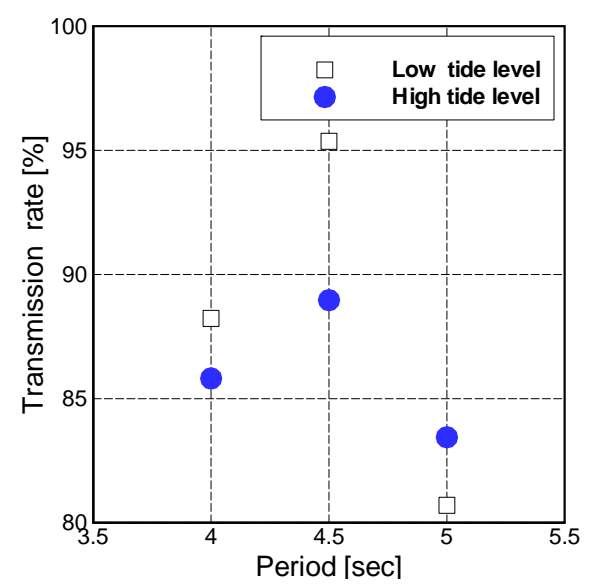

Fig. 13 Transmission rate of floating breakwater according to the wave period. 


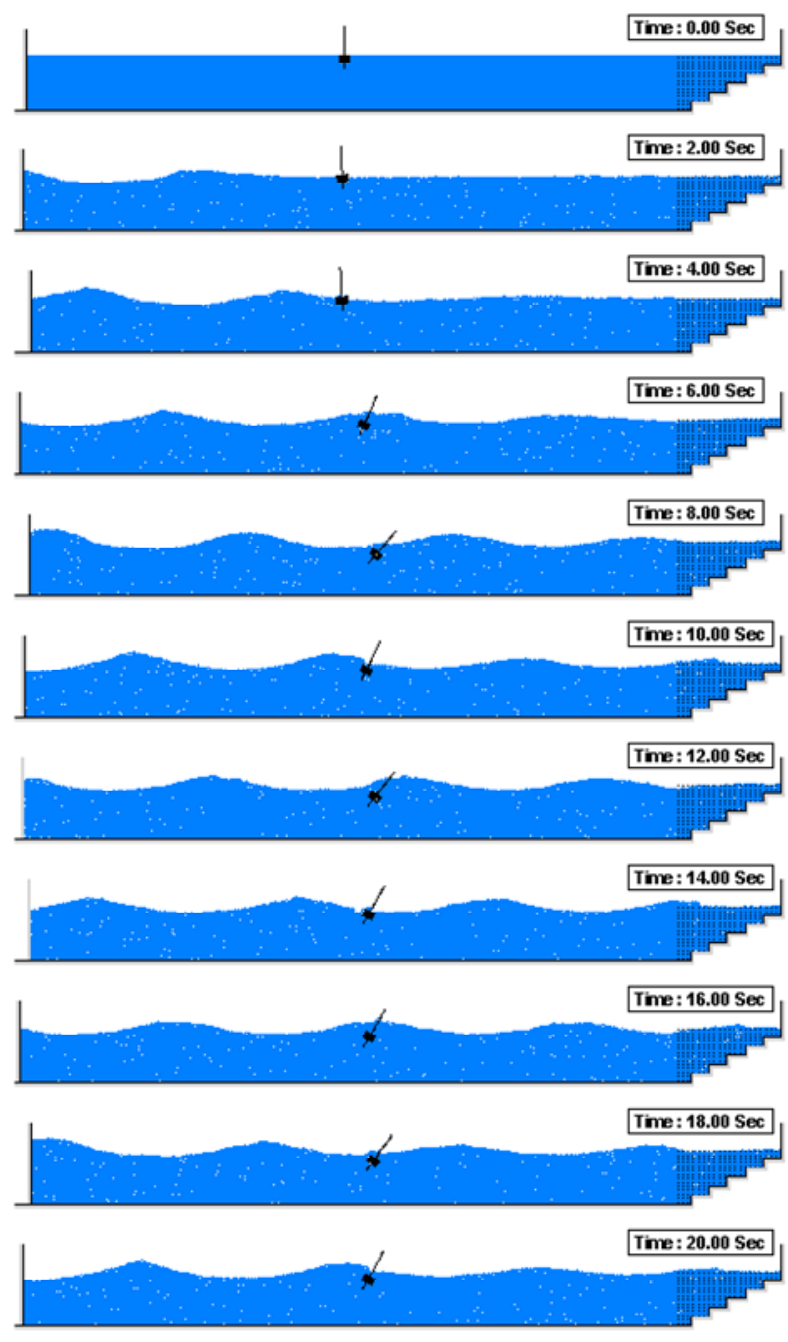

(a) Low tide level
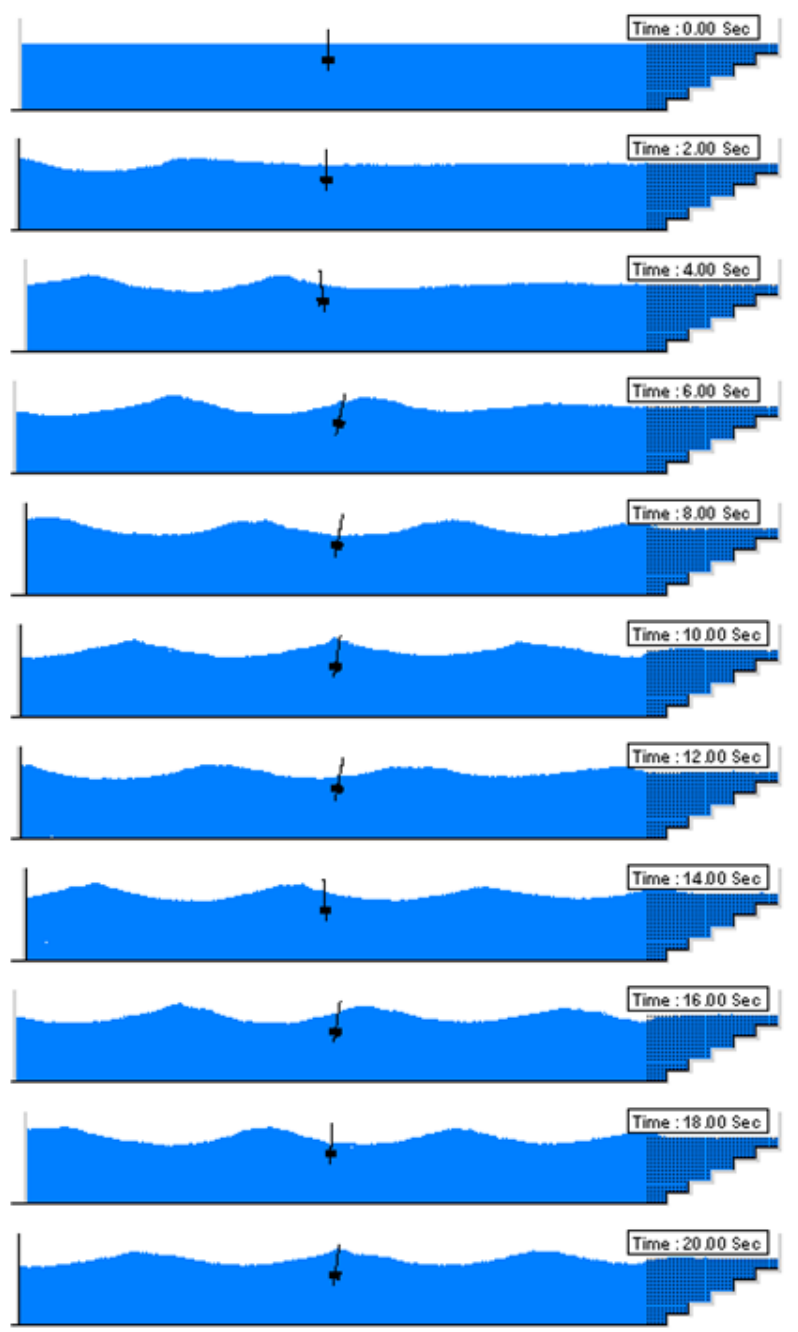

(b) High tide level

Fig.12 Free-surface deformation and motion of floating breakwater interacting with waves (Period=4.5sec, Wave length $=31.62 \mathrm{~m})$.

Fig. 12 shows the free-surface deformation and motion of the floating breakwater interacting with waves. In the case of the low tide level, the breakwater was inclined to the advancing direction of the waves. In the case of the high tide level; however, the breakwater was moved elliptically, based on its initial position.

Fig. 13 depicts the wave transmissivity as a function of the wave period. The wave transmissivity of the floating breakwater was indicated to be higher, at around a period $=4.5 \mathrm{sec}$, and was more effected at the low than high tide level.

\section{Conclusions}

The prediction of the performance of a floating- type breakwater was investigated numerically using the MPS method proposed by Koshizuka and Oka [6] for an incompressible flow.

From the simulated results, the present method appeared to be applicable to the complicated wave motions interacting with a floating body and for the prediction of the transmission coefficients of a floating breakwater with moored lines.

\section{References}

[1] Cho, W.C. and Lee, J.W., Wave Screening Performance Using Floating and Submerged Breakwaters, J. KSCO, 15 (4) (2003) 224-231.

[2] Frederiksen, H.D., Wave attenuation by fluid filled bags, Journal of the Water way, Harbors and coast- 
al Engineering Division ASCE 97 (1971) 73-90.

[3] Hales, L.Z., Floating breakers: State of the Art Literature Review, Technical Report No. 81-1, Coastal Engineering Research Center, US Army Corps of Engineers, Fort Belvoir, VA, (1981).

[4] Idelsohn, S.R., Oñate, E. and Del Pin, F. (2004), The particle finite element method: a powerful tool to solve incompressible flows with free-surfaces and breaking waves, International Journal for Numerical Methods in Engineering, 61 (2004) 964-989.

[5] Kobayashi, N. and Wurjanto, A., Wave transmission over submerged breakwater, Journal of Waterway, Port, Coastal and Ocean Engineering, ASCE, 115 (5) (1989) 662-680.

[6] Koshizuka, S. and Oka, Y., Moving Particle Semiimplicit Method for Fragmentation of Incompressible Flulid, Nucl. Sci. Eng., 123 (1996) 421-434

[7] Koshizuka, S., Atsushi, N. and Oka, Y., Numerical analysis of breaking waves using the moving particles semi- implicit method, Int. J. Math. Fluid, 26 (1998) 751-769.

[8] Liang, N.K, Huang, J.S., and Li, C.F., A Study of Spar Buoy Floating Breakwater, Ocean Engineer- ing, 31 (2004) 43-60.

[9] Losada, I.J., and Patterson M.D., Harmonic generation past a submerged porous step, Coastal Engineering, 31 (1997) 281-304.

[10]Monaghan, J.J, An Introduction to SPH, Comput. Phys. Comn., 48 (1988) 89-96.

[11]Sannasiraj, S.A. Sundar, V. and Sundaravadivelu. R., Mooring forces and motion responses of pontoon-type floating breakwater, Ocean Engineering, 25 (1) (1998) 27-48.

[12] Shashikala, A.P., Sundaravadivleu, P. and Ganapathy, C., Dynamics of a moored barge under regular and random waves, Ocean Engineering, 24 (5) (1977) 401-430.

[13]Sueyoshi, M., Numerical Simulation of Extreme Motions of a Floating Body by MPS Method, MTS/IEEE TECHNO-OCEAN’04, 1 (2004) 566572.

[14]Van der meer, J.W., Deamen, F.R., Stability and wave transmission at low-crested rubble mound structures, Journal of Waterway, Port, Coastal and Ocean Engineering, ASCE, 120 (1) (1994) 1-19. 